\title{
A Study of Multibiometric Traits of Identical Twins
}

\author{
Zhenan $\operatorname{Sun}^{a}$, Alessandra A. Paulino ${ }^{b}$, Jianjiang Feng $^{b}$, Zhenhua Chai $^{a}$, \\ Tieniu Tan ${ }^{a}$, Anil K. Jain ${ }^{b, c}$ \\ ${ }^{a}$ Center for Biometrics and Security Research, National Laboratory of Pattern Recognition, \\ Institute of Automation, Chinese Academy of Sciences, Beijing, 100190, China; \\ ${ }^{b}$ Department of Computer Science and Engineering, Michigan State University, East Lansing, \\ MI 48824, USA \\ ${ }^{c}$ Department of Brain and Cognitive Engineering, Korea University, Anam-dong, Seongbuk-gu, \\ Seoul 136-713, Korea
}

\begin{abstract}
The increase in twin births has created a requirement for biometric systems to accurately determine the identity of a person who has an identical twin. The discriminability of some of the identical twin biometric traits, such as fingerprints, iris, and palmprints, is supported by anatomy and the formation process of the biometric characteristic, which state they are different even in identical twins due to a number of random factors during the gestation period. For the first time, we collected multiple biometric traits (fingerprint, face, and iris) of 66 families of twins, and we performed unimodal and multimodal matching experiments to assess the ability of biometric systems in distinguishing identical twins. Our experiments show that unimodal finger biometric systems can distinguish two different persons who are not identical twins better than they can distinguish identical twins; this difference is much larger in the face biometric system and it is not significant in the iris biometric system. Multimodal biometric systems that combine different units of the same biometric modality (e.g. multiple fingerprints or left and right irises*) show the best performance among all the unimodal and multimodal biometric systems, achieving an almost perfect separation between genuine and impostor distributions.
\end{abstract}

Keywords: Identical twins, biometrics, fingerprint, iris, face

\section{INTRODUCTION}

In the year 2005, approximately 161 of every 100,000 births in the United States were triplets or more. This birth rate increased $400 \%$ during the 1980s and 1990s, and then started to slowly decline after 1998. The increase of multiple births in the last few decades has been associated with the increase in the use of fertility therapies and "the older age at childbearing", the latter because "women in their 30s are more likely than younger women to conceive multiples spontaneously." The twin birth rate (32.2 per 1000 births) increased at an average rate of $3 \%$ per year between 1990 and 2004. Thus, the total increase in twin births since 1990 is $42 \%$ and since 1980, $70 \%{ }^{1}$ This, in turn, has created a greater demand for biometric identification systems that can accurately determine the identity of a person who has an identical twin.

Accurately distinguishing the identity of twins has important legal ramifications. In 1985, a woman was accused of making fraudulent money transfers from banks nationwide by simply making telephone calls. She was acquitted of those charges after claiming her sister - who was her identical twin but disappeared before the trial - committed the crimes. In the end, both twins were convicted. Based on an analysis of their voices,

Further author information: (Send correspondence to A.A.P.)

Z.S.: E-mail: zhenan.sun@ia.ac.cn, Telephone: 861082610278

A.A.P.: E-mail: paulinoa@cse.msu.edu, Telephone: 15173559319

J.F.: E-mail: jfeng@cse.msu.edu, Telephone: 15173559319

Z.C.: E-mail: zhchai@nlpr.ia.ac.cn, Telephone: 861082621145

T.T.: E-mail: tnt@nlpr.ia.ac.cn, Telephone: 861082614515

A.K.J.: E-mail: jain@cse.msu.edu, Telephone: 15173559282

${ }^{*}$ Some dictionaries define irides as the plural form of iris in the context of the part of the eye. 
a speech scientist and voice examiner testified that both women had placed the fraudulent telephone calls. ${ }^{2}$ In this case, being able to distinguish both twins was essential to the convictions.

More recently, a rape suspect won two mistrials because there was no way of determining whether the key DNA evidence came from him or his identical twin. However, in his third trial in 2006, he was convicted after the prosecutors provided evidence that he had committed a series of sexual assaults and attempted sexual assaults with characteristics similar to the rape for which he was being tried then. ${ }^{3}$ Therefore, the failure to distinguish the twins was crucial to the charges being dropped in the first two mistrials.

The similar appearances of identical twins may also give them a greater incentive to commit fraud than average persons. Imagine a scenario where you have an identical twin brother/sister, and he/she has health insurance but you do not, and you get sick. In order to get medical treatment, the health insurance company requires health providers to establish your identity using a photo identification. You could use your twin's health insurance without being noticed. But, let us suppose the health insurance company requires that you need to be identified using fingerprints (this is the case for some of the health insurance companies in Brazil); if the fingerprint recognition system can distinguish twin fingerprints, then you will not be able to get health care using your twin's name and insurance policy.

The above facts and scenario indicate that the ability of biometric systems to identify identical twins is necessary. There are two types of twins: monozygotic (or identical) and dizygotic (or non-identical). Monozygotic twins are a result of a single fertilized egg that splits into two cells, each one giving origin to one individual. Dizygotic twins are a result of two different fertilized eggs. Monozygotic twins have the same deoxyribonucleic acid (DNA) and, therefore, they cannot be distinguished using DNA. ${ }^{4}$ Thus, it is necessary to use other forms of identification for monozygotic twins.

Recognition using biometric traits is now a well accepted and proven method. A biometric characteristic is a detectable biological or behavioral characteristic of an individual that is distinguishable and repeatable. Some examples include fingerprints, face, palmprints, iris, retina, and voice. A biometric system relies on the distinctiveness of the biometric characteristics to perform the recognition. While many biometric techniques are extremely accurate, some variations in sensing data, noise, etc. can cause the system performance to drop significantly. We could say it is more difficult to discriminate identical twins than unrelated persons because of their genetic similarity. Although identical twins cannot be distinguished from each other using DNA, some of the biometric modalities, such as fingerprints, iris, and palmprints, can still be used to distinguish them. ${ }^{5}$ Some experiments show that face and voice ${ }^{6,7}$ can be used to distinguish identical twins. Due to the difficulty in obtaining a large biometric database of identical twins, most experiments are performed on small databases, making the conclusions less reliable.

This paper explores the impact of identical twins on unimodal and multimodal biometric systems. In Section 2 , we present previous studies based on biometric twin data. In Section 3, we present the biology of the three biometric characteristics that will be used in our multibiometric experiments, namely fingerprint, face, and iris. In Section 4, we analyze the experimental results on matching individual biometric traits as well as various combinations of modalities. In Section 5, we present our conclusions.

\section{BACKGROUND}

In order to design a robust and efficient biometric system, the system must be able to handle a variety of situations like noisy data, limitations of the sensors, environmental conditions, and the presence of identical twins. Due to the similarity of their biometric characteristics, identical twins are more likely to pose a challenge to a biometric system. Therefore, it is important to address this problem when designing a biometric system. Studies on discrimination of identical twins that have been reported in the literature are summarized below.

Daugman and Downing ${ }^{8}$ assessed the distinctiveness of iris patterns as biometric identifiers. The authors compared unrelated irises (irises from different persons), and genetically identical irises (irises that came from the same DNA), for example pair of irises of the same person or the irises of identical twins. They observed that the iris patterns of genetically identical eyes were as uncorrelated as the patterns of unrelated eyes. For example, the similarity of the six pairwise comparisons they performed between identical twins showed the same mean as for eyes that were not genetically related. 
In a study of identical twins' palmprints, Kong et al. ${ }^{5}$ used 1,028 palmprint images from 53 pairs of identical twins. They performed two different twin matches. In the first experiment, they matched the palmprints of identical twins, which they called real twin match. In the second experiment, they matched the left and right palmprints of the same person, which they called virtual twin match. Note that in both the experiments the palmprints shared the same genetic information. The authors observed that while these genetically equivalent palmprints have correlated features, they can still be distinguished based on non-genetically related information.

The above observation concerning palmprints is also true for fingerprints, as a study based on 94 pairs of identical twin fingerprints has shown. ${ }^{4}$ Fingerprint verification systems can be used to distinguish identical twins, even though their fingerprints are generally more correlated than fingerprints coming from two genetically unrelated persons. As an example, at a False Rejection Rate (FRR) of $1.05 \%$, the twin-twin matching had a False Acceptance Rate (FAR) of $8.51 \%$, while the twin-nontwin matching had a FAR of $2.20 \%$. For another threshold value (FRR of $3.49 \%$ ), the twin-twin FAR dropped to $1.06 \%$ and the twin-nontwin FAR dropped to $0.29 \%$. In another analysis of fingerprints from 66 pairs of twins, ${ }^{9}$ it was also concluded that fingerprints can be used to identify identical twins with an insignificant drop in the performance: the Equal Error Rate (EER) reportedly increased by only 1-2\% compared to nontwin impostor matchings. The authors also extended their studies to assess the similarities among families of nontwins (52 families of four persons - parents and two children). They observed that the largest similarity occurred between identical twins, followed by between two siblings, between parents and their children, and between unrelated persons.

Srihari et al. analyzed the similarity between twins' fingerprints in a study using fingerprint images from 298 pairs of twins. ${ }^{10}$ The authors analyzed this similarity based on Level 1 and Level 2 features (that is, pattern of the ridge flow, and minutiae, respectively). With the level 1 features, they observed that fingers with the same label twins' fingers occurred approximately $55 \%$ of the time for twins and approximately $32 \%$ for non-twins, which means twins' fingers are much more likely to have the same pattern type than non-twins' fingers. With the level 2 features, they concluded that the similarity between twin fingers is higher than between two arbitrary fingers (with identical twin fingers similarity being not significantly different from fraternal one), but twins can still be distinguished using fingerprints.

Although it is believed that face and voice, along with hand geometry, are more difficult to distinguish between identical twins ${ }^{4}$ some researchers have obtained encouraging results using face and voice to distinguish monozygotic twins. ${ }^{6,7,11}$ A verification experiment with 10 sets of identical twins was performed using a 2D face recognition system; the experiment consisted of enrolling one of the twins, and asking the other to try to log into the system. On this small database, the rejection threshold was always satisfied, leading to a rejection of all the impostor twins. ${ }^{6}$

Another face recognition experiment for twins was based on 3D facial images. ${ }^{11}$ The recognition task was to distinguish between two identical twins; the authors tested three different algorithms: a $2 \mathrm{D}$ algorithm based on eigenfaces, a 3D algorithm based on rigid surface, and another $3 \mathrm{D}$ algorithm based on canonical forms. The test consisted of enrolling one of the twins and matching the enrolled subject to the other twin. All the other subjects served as impostors. For the first algorithm (2D eigenfaces), the False Acceptance Rate (FAR) was $29.41 \%$ when enrolling the first twin and $25 \%$ when enrolling the other. In the second experiment, the FAR was $17.64 \%$ and 0 , respectively, while for the third algorithm perfect matchings were obtained. According to the Web site Digital World Tokyo, ${ }^{12}$ the Japanese company Sagawa Advance has invented an infra-red based face recognition technology that is able to distinguish identical twins.

Ariyaeeinia et al. ${ }^{7}$ performed recognition experiments using speech data from 49 pairs of identical twins. The authors performed basically two different experiments: an general experiment, in which any two persons in the dataset were considered impostors, and the twin experiment, in which the impostor tests consisted of the tests between a person and his/her twin. The Equal Error Rate reported was 1.0\% for the twin experiment using short test data (each person saying his/her date of birth), and $0.5 \%$ for the general configuration. Other authors have tried to distinguish identical twins based on voice in a multilingual environment. ${ }^{13}$ Using a database of 12 twins, Patil and Basu reported the highest success rate as being $100 \%$ for a particular size (in seconds) of the training speech, and particular size (in seconds) of the test speech. They also observed that the majority of errors were due to matching the actual speaker with his/her twin brother/sister. 
However, in many previous studies, the identical twin biometric databases are very small and in-house biometric matchers instead of the state-of-the-art matchers are used. This affects the reliability of conclusions. Moreover, no study has been conducted to compare and fuse multiple biometric traits of identical twins.

\section{MULTIBIOMETRICS}

A multibiometric system uses multiple sources of biometric information in order to recognize an individual. For example, a multibiometric system may use fingerprint and face, the left and right iris, or a fusion of different fingerprint matching algorithms to recognize a person. In the next subsections, we focus our attention on the distinctiveness of fingerprints, face, and iris for identical twins. These three modalities will be used in our multibiometric experiments.

\subsection{Fingerprint Discriminability}

A fingerprint is the impression of the friction skin on a finger. The individual characteristics of friction ridge skin are determined during fetal development. Their formation is similar to the formation of blood vessels or capillaries during the growth of the fetus in the uterus. The fingerprint formation starts at approximately 6 or 7 weeks of gestational age and it is due to the flow of amniotic fluids in a micro-environment. ${ }^{14} \mathrm{~A}$ minor change in this flow and in the position of the fetus in the uterus cause the minute skin structures around palm or finger tips to differentiate. Friction ridge skin can be distinguished from the skin of the rest of the body due to a variety of factors, such as the presence of raised ridges, increased sensory abilities, absence of hair and sebaceous glands, and a thicker and more complex epidermis. Friction ridges are related to grasping and gripping, which explains their presence in our hands and feet.

A fingerprint pattern has some details inherent to each individual, like ridge endings, the point where a ridge ends abruptly, or a ridge bifurcation or trifurcation, where the ridges are divided into different branches. These details are called minutiae points and they are supposedly different in every fingerprint, even in prints of identical twins, because, as mentioned before, a very small difference in micro-environment is sufficient to change the process of cell formation, causing minutiae points to be different. As a result, fingerprint is considered very reliable in terms of biometric identification because of its distinctiveness. Another reason for this reliability is that fingerprints do not change significantly over time, an essential characteristic of a biometric modality since a biometric system is typically meant to be used to identify a person over a long period of time.

A friction ridge pattern consists of three levels of detail, which are used for fingerprint matching: ridge flow and pattern (level 1 features), ridge path and minutiae (level 2 features), and dimensional, edge shape and pore details within a specific ridge (level 3 features). Ridge flow pattern falls into three general categories: whorls, in which the ridge flows form a complete circuit; loops, in which the ridge flow enters from one side of the fingerprint, curves and returns in the same direction from which it came; and arches, in which the ridge flow enters from one side and exits the opposite side. In the second level features, ridges vary in length, and can be dots, which are very short ridges containing just one pore, or they can cross the entire area of friction skin without being broken. An example of the third level feature is the sweat pores that are regularly spaced along the ridges and whose specific locations and shape can be used as distinctive features for identification. ${ }^{14}$

\subsection{Face Discriminability}

Face is composed of the skull characteristics and the musculature and associated soft tissue. To study the variation among human faces it is necessary to study these structures. The facial skeleton serves as the bony framework for the mimetic musculature. Since these muscles are stretched across the facial skeleton like a mask, the variation in facial appearance is caused mostly because of the form of the facial bones. A majority of individuals can be divided in three categories in terms of facial appearance: (i) a long, narrow head, (ii) a proportional length to width head and (iii) a short, wide head; these differences in facial shapes are due to the form of the cranial base. ${ }^{14}$

Facial form is influenced by gender. Males are more likely to have larger faces because of their usually larger bodies and their need of more air in order to support larger muscles and visceras, causing them to also have a larger nose. Males usually have a more protrusive forehead. Besides the gender influences in the face of an 
individual, a face also changes when a person ages. Infant faces tends to be wide and short because of the development of the brain; over time, the face develops and this wide and short face tends to change. Other effects of aging are dehydration of the dermis and reabsorption of subcutaneous fat deposits, which result in a decrease in the facial volume and wrinkling. ${ }^{14}$

The muscles may vary in their presence (not everyone has all the muscles that could be in a face), form, location, and control. These factors influence the kind of facial movement that an individual can create. Furthermore, the facial movements of an individual change his/her face as he/she ages. With aging, the elasticity of the skin decreases and the face then is marked with the expressions that occur frequently, becoming relatively permanent features. This fact may explain why identical twin faces are more likely to be distinguished as they are older than when they are infants. ${ }^{14}$

There are a number of factors that influence the performance of a facial recognition system. Besides those already cited above, there are differences in pose, illumination, expression, occlusion, accessories like glasses, weight changes, hair style changes, etc. All these variations make facial recognition systems not as accurate as some other biometrics, like fingerprints. Identical twins present a very difficult situation for face recognition systems, since they are usually very similar in facial appearance.

\subsection{Iris Discriminability}

The iris is an annular shaped part of the eye between the pupil and the sclera that regulates the amount of light entering the eye through the pupil. The iris is physically small in size (about $11 \mathrm{~mm}$ ) but well-designed optical systems can magnify a human iris into a high-resolution image that is 200 to 300 pixels in diameter. There are many minute features such as freckles, coronas, stripes, furrows and crypts, etc. randomly distributed in the iris region, which constitute the unique iris texture for each eye. It should be noted that the visual pattern of a human iris includes both color and texture. However, iris color has limited discriminating power for recognition. So gray-level iris images captured under near infrared illumination are often used to record iris texture pattern for person identification.

The iris texture pattern is formed and becomes stable after the eighth month of gestation. It is commonly believed that the formation of iris pattern is determined by the gestation environment, i.e. iris is a phenotypic biometric trait. ${ }^{8}$ So even identical twins can be discriminated using suitable iris features; even the iris of left and right eyes of the same person are different.

Random processes involved in iris development determine the irregular shape and random spatial layout of micro anatomical structures (MAS). The MAS in the iris surface may exhibit different reflectance properties in near infrared light, leading to sharp intensity variations across iris image region. We can regard each iris region as a piece of $2 \mathrm{D}$ surface in a $3 \mathrm{D}$ coordinate system. So the surface shape from valley to peak resembles an odd Gabor filter and the shape from valley to peak then valley resembles an even Gabor filter, and vice versa. Daugman ${ }^{8}$ proposed to match each iris region with even or odd Gabor filters and then encode positive correlation as 1 and negative correlation as 0 . This way, an iris image can be represented by 256 bytes, called the iris code. The iris codes of twins (or non-twins) have only $50 \%$ chance of being matched because their corresponding regions independently have an equal probability to be either 1 or 0 in iris feature coding. In contrast, multiple iris images of the same eye have a much higher probability than $50 \%$ to be matched in their iris codes, even though noise may perturb some parts of iris codes. A more general explanation of the effectiveness of iris recognition for twins is based on ordinal measures. ${ }^{15}$

\section{EXPERIMENTAL RESULTS AND ANALYSIS}

The first version of the CASIA Multimodal Biometrics Database of Twins (CASIA-TwinsV1) was collected on October 2, 2007 at the Beijing Chaoyang Park during the Fourth Annual Festival of Beijing Twins Day. Figure 1(a) shows the kiosk where the biometric acquisition was performed and Figure 1(b) shows the face acquisition device. This database includes face, iris, and fingerprint images from 92 pairs of twins and 2 sets of triplets. All the images were captured indoors on the same day (i. e., single data capture session).

Not all of the 94 families provided images for all the modalities. Since some of our experiments involve combinations of units/modalities, we considered only those individuals who have a complete set of images (face, 


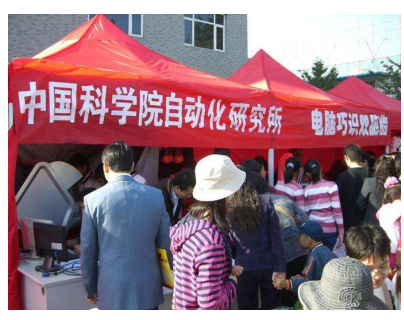

(a)

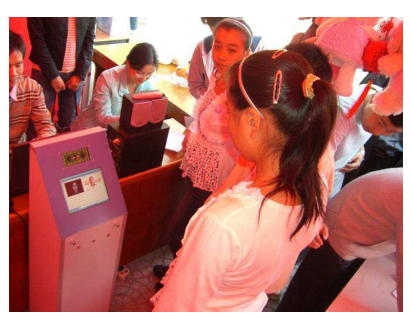

(b)

Figure 1. The kiosk for biometric acquisition (a) and the face acquisition device (b).

two irises and four fingerprints) in the database, and whose twin's images were also present in the database as a complete set. As a result, the total number of subjects used in our experiments consisted of 134 subjects (66 families, including two families of triplets - 51 pairs of identical twins and 15 pairs of non-identical twins). For all units (four fingers, two irises, and face), the number of genuine matches performed was 134, the number of identical twin impostor matches was 102, and the number of general impostor matches was 17,720.

Most of the twins are identical (or monozygotic twins), but some are non-identical (or dizygotic) twins. This information was not recorded, so we derived this information based on observing whether the facial images of a set of twins were very similar or not ${ }^{\dagger}$. We divided our database into two groups, identical twins and non-identical twins. Most of the subjects in the database are children, but there are some adults as well. The subject age ranges from 5 to 65 , with the average age being 16.8. In the following sections, we present detailed descriptions of the databases used, the experiments performed, and experimental results.

\subsection{Fingerprint}

The fingerprint images were captured using Symwave sw6888, ${ }^{16}$ a sweep sensor, with a resolution of 500 dpi. This dataset contains images from four different fingers and the number of images per finger is not fixed, but varies from 6 to 31. Because of this large variability and the poor quality of many images, we selected one image per finger as the template and another image from the same finger as the query image that contain the largest number of minutiae.

Figure 2 shows some fingerprint images from the twin dataset. Figure 2(a) shows images of the four fingers $1,2,3$, and 4 for the first twins of an identical twin pair; Figure 2(b) shows the fingerprints of the corresponding fingers for the second twins of the pair. Figures 2(c) and 2(d) follow the same scheme for a non-identical twin pair. The similarity between ridge flow patterns of corresponding fingers of identical twins is evident. While all four pairs of corresponding fingers of identical twins have the same fingerprint pattern type, only two pairs of corresponding fingers of non-identical twins have the same pattern type.

A commercial minutiae based matcher, VeriFinger, ${ }^{17}$ was used to obtain the matching score in the fingerprint experiments.

Three distributions were generated in order to observe the results of the experiments. The genuine distribution was obtained by matching the gallery image of one modality of one individual to the probe image of the same modality and the same individual. Identical twin impostor distribution was generated by matching each image of one person to his/her identical twin. The general impostor distribution was generated by all the impostor matches except the identical impostor matches described above. This scheme was used for all the experiments.

Figures 5(a) and 5(b) show the genuine, identical twin, and the general impostor distributions of finger 3, which had the worst performance among the four fingers, and finger 4, the best performing finger, respectively. We can see that, although identical twin impostor distribution of finger no. 3 is similar to the general impostor distribution of this same finger, there are some peaks in the right tail of the identical twin impostor distribution that differentiates them. This is an indicator of the larger similarity between fingerprints of identical twins compared to the similarity between fingerprints of unrelated persons. This is also the case for finger no. 4. Another indicator of this larger similarity is the number of matched minutiae. For example for finger 4, the

\footnotetext{
${ }^{\dagger}$ The ideal method to distinguish identical or non-identical twins should be based on DNA.
} 

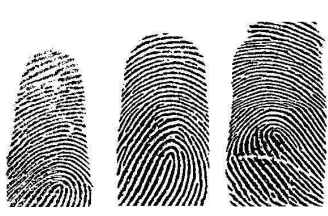

(a)
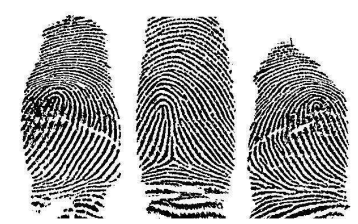

(c)
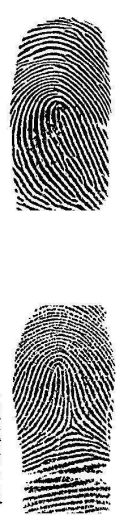

Figure 2. Fingerprint images of fingers 1, 2, 3, and 4 of the first twin (a), and the four images of the corresponding fingers of an identical twin pair (b); similarly, (c) and (d) show fingerprint images of a non-identical twin pair. Note the similarity in ridge flow pattern between identical twins. All four corresponding fingers of identical twins in (a) and (b) have the same pattern. But for non-identical twins in (c) and (d), only two corresponding fingers (no. 1 and 3) have the same pattern.

mean of the number of matched minutiae for genuine pairs is $22.00( \pm 9.50)$; for identical twin pairs, $6.44( \pm$ $3.97)$; and for general impostor pairs, $4.22( \pm 2.82)$. For the other three fingers, those numbers are about the same. Although the number of matched minutiae for identical twin pairs is much smaller than for genuine pairs, it is still larger than the number of matched minutiae for general impostor pairs, which indicates the similarity between identical twins' fingerprints is larger than between unrelated persons.

\subsection{Face}

The facial images were captured in color, all of them from a USB camera. The image size is $480 \times 640$, but the face area varies from $280 \times 300$ to $300 \times 400$. The images contain non-uniform background, and some variations in illumination. The images were captured in a sequence and over a short time interval. There are about 20 face images per subject. Some facial image examples are shown in Figure 3. The first two images show facial images of an identical twin pair (Figure 3(a)), while the other two are facial images of a non-identical twin pair (Figure $3(\mathrm{~b}))$.

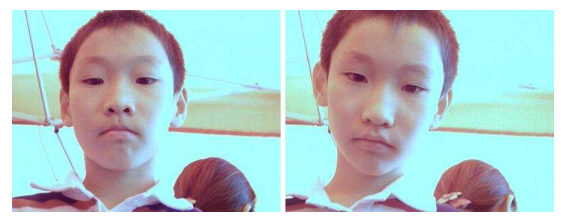

(a)

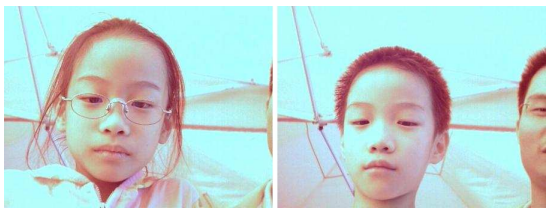

(b)

Figure 3. Facial images of the first and second twin in an identical twin pair (a), and a non-identical twin pair (b).

The face subset used in the experiments contained 134 subjects, each one having around 20 images. The commercial matcher used to perform the facial matchings was FaceVACS. ${ }^{18}$ We considered two facial images per person (the template and the query) in our experiment, mainly because the pictures were taken over a very short time interval, which makes them very similar. The first and the last images of each person were used, since they are expected to be the least similar. Figure 5(c) shows identical twin and general impostor distributions, along with the genuine distribution for the face experiments. In the genuine distribution, we can see that there is almost no tail and that about $90 \%$ of the genuine matches has a score of more than 0.95 . This is due to the high similarity between the two images of the same person, since they were taken in sequence and in a very short time interval. Also, we can observe that the identical twin impostor distribution is much more similar to the 
genuine distribution than to the general impostor distributions, meaning identical twins are a real challenge to face recognition systems.

\subsection{Iris}

The iris images were captured using a IKEMB-100 camera produced by IrisKing. ${ }^{19}$ The size of the images is $480 \times 640$, but the approximate iris diameter is 200 pixels. It is an embedded dual-eye iris camera and has a LCD displaying real time iris images for user convenience. The iris images were captured in sequence and over a short time interval. There are 10 images for most of the subjects, with a few individuals having a smaller number of images. Some examples of iris images are shown in Figure 4. Figures 4(a) and 4(b) show iris images of an identical twin pair, where the two images in 4(a) are the left and right iris images of the first twin, and the two images in 4(b) are the left an right iris images of the second twin. Similarly, Figures 4(c) and 4(d) show iris images of a non-identical twin pair.

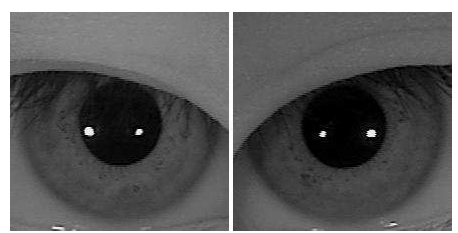

(a)

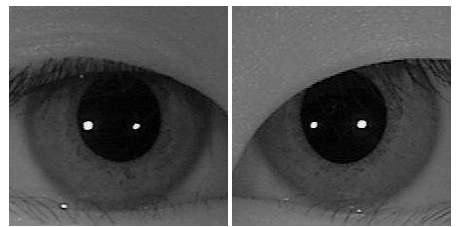

(c)

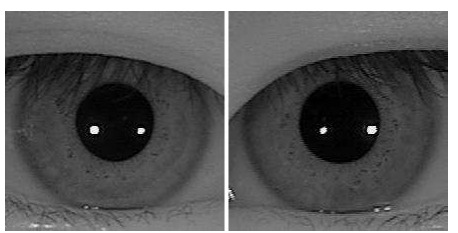

(b)

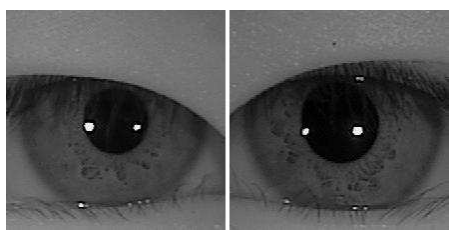

(d)

Figure 4. The left and right iris images of identical ((a) and (b)) and non-identical twin pairs ((c) and (d)).

The iris feature representation method based on ordinal measures ${ }^{15}$ is used to test the performance of iris recognition for twins. Two iris images were randomly selected as probe and gallery for each eye. Figure 5(d) shows the genuine, identical twin, and general impostor distributions of the right iris, which performed slightly better than the left iris. The identical twin impostor distribution is very similar to the general impostor distribution. However, the peaks that are present in the identical twin impostor distribution tail may indicate that the irises of identical twins have some correlation.

\subsection{Multibiometric Experimental Results}

In our multibiometric experiments, we first combined units of the same modality, like the two irises or all the four fingers of a person. We performed a simple fusion using scores from the four fingers by summing them. The identical twin and general impostor distributions, along with the genuine distributions, are shown in Figure 6(a). A fusion using both the irises was also performed, and the distributions are shown in Figure 6(b). We can observe from these figures that the matching results are extremely good; genuine and impostor distributions are well-separated. This indicates that multimodal biometric systems can work very well even in the presence of identical twins in the biometric database.

Two different Receiver Operating Characteristic (ROC) curves were generated for each experiment. Identical twin impostor ROC curve means the impostor matches considered were just identical twin impostor matches, while a general impostor ROC curve means we considered all the impostor matches, except the identical twin impostor matches. Figure 7(a) shows the ROC curves for fingers nos. 3 and 4 , the best and worst performing fingers, respectively, and the ROC curves of the 4-finger fusion. The performance of the fingerprint identification system in distinguishing genuine matches from general impostor matches is always better than the performance in distinguishing genuine matches from identical twin impostor matches. It is important to note that the 4-finger fusion had the highest performance among the experiments involving fingerprints. 


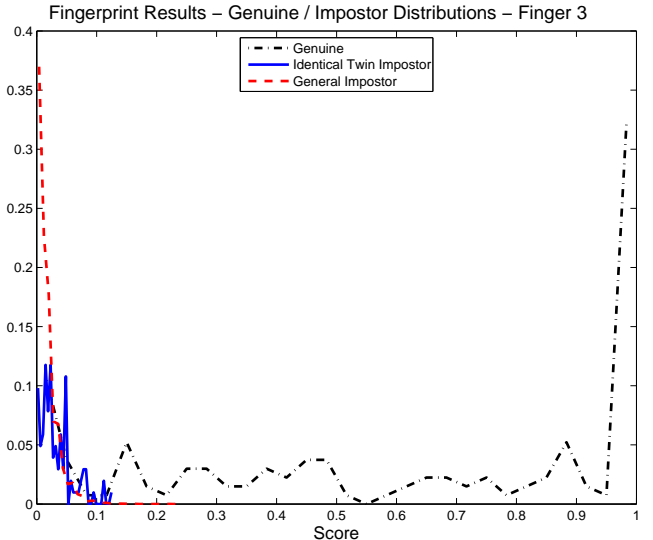

(a) Finger 3

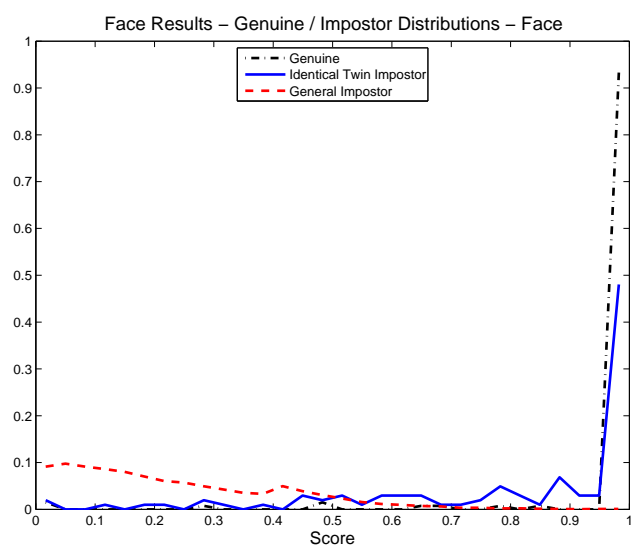

(c) Face

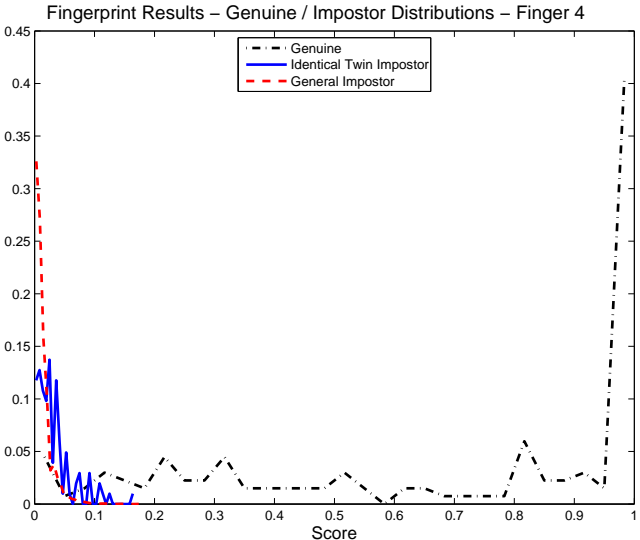

(b) Finger 4

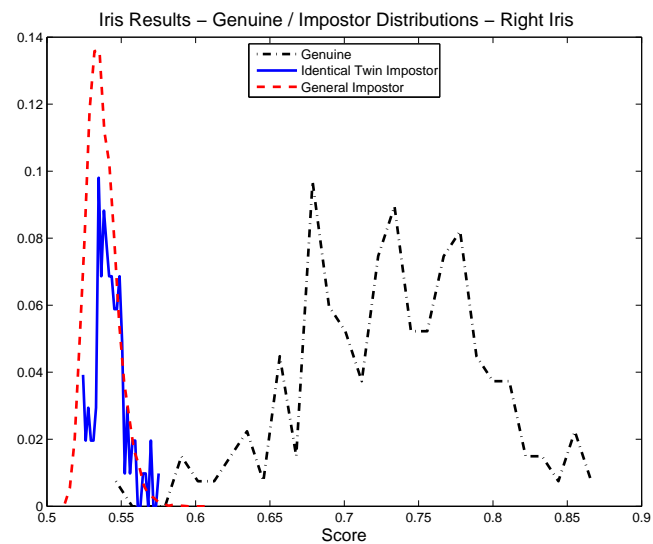

(d) Right Iris

Figure 5. Identical twin, general impostor distributions and genuine distributions for fingers 3 and 4, face, and right iris.

The iris matching experiment results show that the iris biometric system can distinguish identical twins as much as it can distinguish any two different persons who are not identical twins, as shown in Figure 7(b). Iris experiments presented the best performance among all the experiments that used just one biometric characteristic (one finger, one iris, or face). This may be because irises from identical twins are more uncorrelated than fingerprints from identical twins, or may be due to the fact that iris images in our dataset have a very good quality due to automatic rejection of poor quality images by quality control software, while the quality of many fingerprint images is very poor due to improper sweep operation by some of the children, and the image area is very small. Also, the 2-iris fusion showed improvements compared to the performance of each iris alone.

We also fused face and finger no. 4, where the fingerprint scores were appropriately normalized, and the results are shown in Figure 7(d). We did not combine iris with another biometric modality because iris results are already really good and the database is not large enough for measuring lower error rate. The ROC curves of the face experiments are shown in Figure 7(c). The face experiment shows that the presence of identical twins in the face database causes the face recognition performance to drop. Although the performance was good based on the general impostors (no identical twins in the data involved), we can only get a True Acceptance Rate (TAR) for the identical twin data greater than zero at a false acceptance rate of over $10 \%$ for identical twins. Compared to face matcher, the fusion of face and finger no. 4 has a better performance in dealing with identical twin and general impostors. Compared to fingerprint matcher, this fusion degraded the performance on identical twin impostors, but improved the performance on general impostors. It should be noted that the inferior performance of fingerprint matcher compared to face matcher in our experiments is due to the specific fingerprint sensor (swipe sensor) used for data collection and the difficulty of obtaining good quality fingerprint 


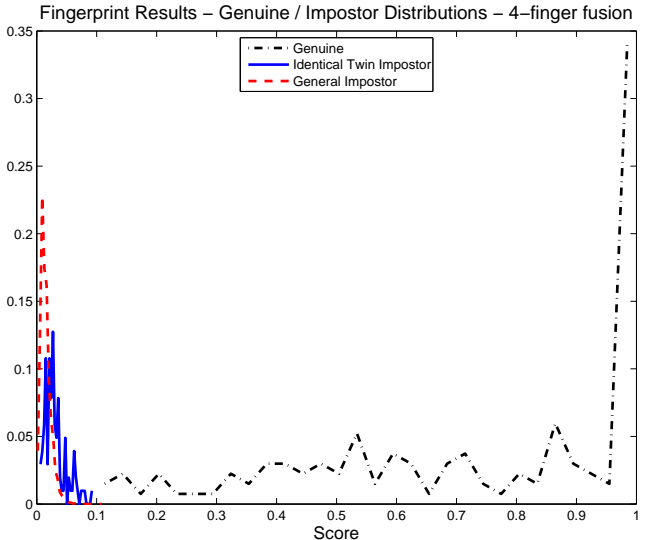

(a) 4-finger fusion

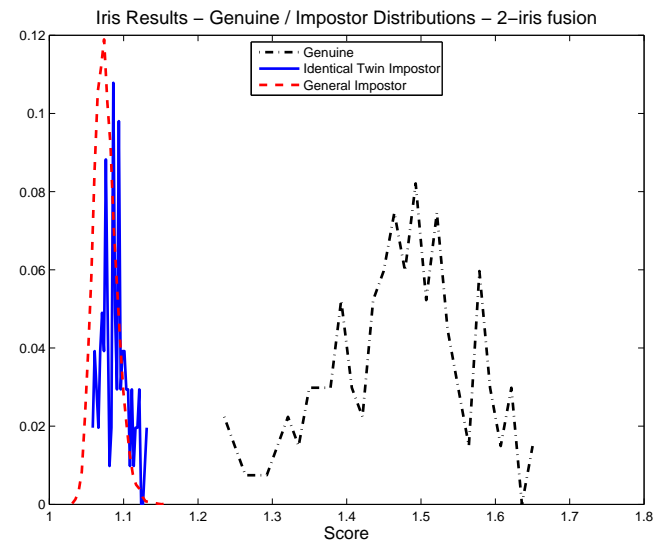

(b) 2-iris fusion

Figure 6. Identical twin, general impostor, and genuine distributions of the 4-finger fusion and 2-iris fusion, respectively.

images of younger subjects using the swipe sensor. On the other hand, there is very small intraclass variation for face images.

Table 1 shows the equal error rates for the worst and best performing fingers (finger numbers 3 and 4 ), 4 -finger fusion, left and right irises, iris fusion, face, and face and finger 4 fusion; the equal error rates are shown based on the identical twin impostors and general impostors separately. It can be observed that all the modalities have a better performance on the general population than on the identical twin population.

Table 1. Equal Error Rate (\%) for the experiments, based on identical twins and on the general impostors.

\begin{tabular}{|l|c|c|c|c|c|c|c|c|}
\hline Modality & $\begin{array}{c}\text { Finger } \\
3\end{array}$ & $\begin{array}{c}\text { Finger } \\
4\end{array}$ & $\begin{array}{c}\text { 4-finger } \\
\text { fusion }\end{array}$ & $\begin{array}{c}\text { Left } \\
\text { Iris }\end{array}$ & $\begin{array}{c}\text { Right } \\
\text { Iris }\end{array}$ & $\begin{array}{c}\text { 2-iris } \\
\text { fusion }\end{array}$ & Face & $\begin{array}{c}\text { Face+ } \\
\text { Finger 4 }\end{array}$ \\
\hline Identical twins & 13.95 & 6.79 & $\mathbf{0 . 4 9}$ & 1.35 & 0.86 & $\mathbf{0 . 4 9}$ & 13.67 & 7.65 \\
\hline General impostors & 10.71 & 4.40 & $\mathbf{0 . 0 0}$ & 0.75 & 0.75 & $\mathbf{0 . 0 0}$ & 3.79 & 2.48 \\
\hline
\end{tabular}

\section{CONCLUSIONS}

We have presented a study of the distinctiveness of biometric characteristics of identical twins (fingerprint, iris and face). The discriminability of these three biometric traits is supported by anatomy and the formation process of the biometric characteristics, as discussed in Section 3. We assessed the capacity of existing commercial biometric matchers in distinguishing identical twins based on fingerprint, iris, and face.

The unimodal face biometric system can distinguish two different persons who are not identical twins much better than it can distinguish identical twins. Although the unimodal fingerprint biometric system also can discriminate two different persons who are not identical twins better than it can discriminate identical twins, this difference is not as large as for the face biometric system. The iris matching experiment results show that there is no significant difference in the performance of the biometric system for the identical twin data and for the general data, which means the iris biometric system can distinguish identical twins to the same extent as it can distinguish any two persons who are not identical twins. Among the unimodal biometric systems, left and right iris performed the best. Again, this may be due to the fact that irises from identical twins are more uncorrelated than fingerprints from identical twins, or may be due to the fact that iris images in our dataset have a very good quality, while the quality of many fingerprint images is very poor and the image area is very small.

Multimodal biometric systems that combine different units of the same biometric modality (4-finger and 2-iris fusions) lead to an almost perfect separation between genuine and impostor distributions. For both general 


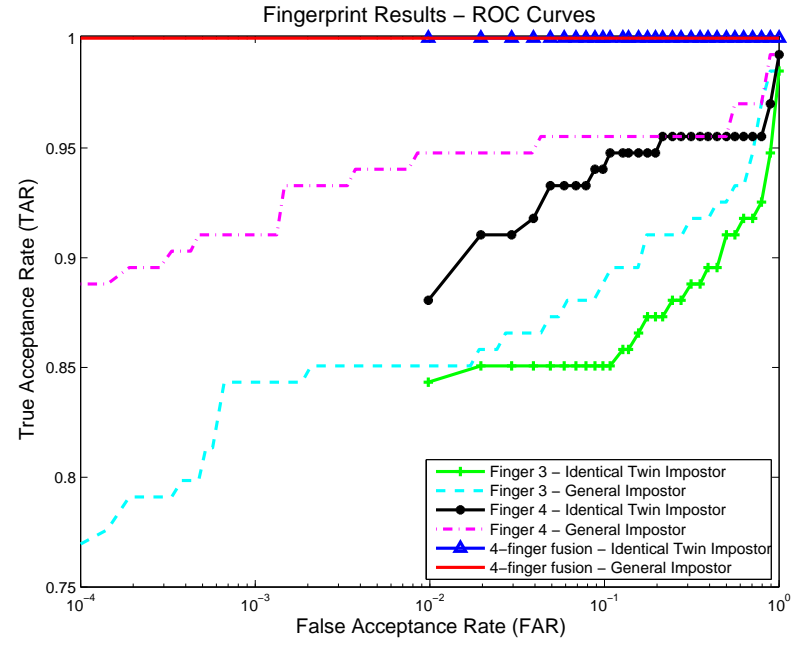

(a) Fingerprint

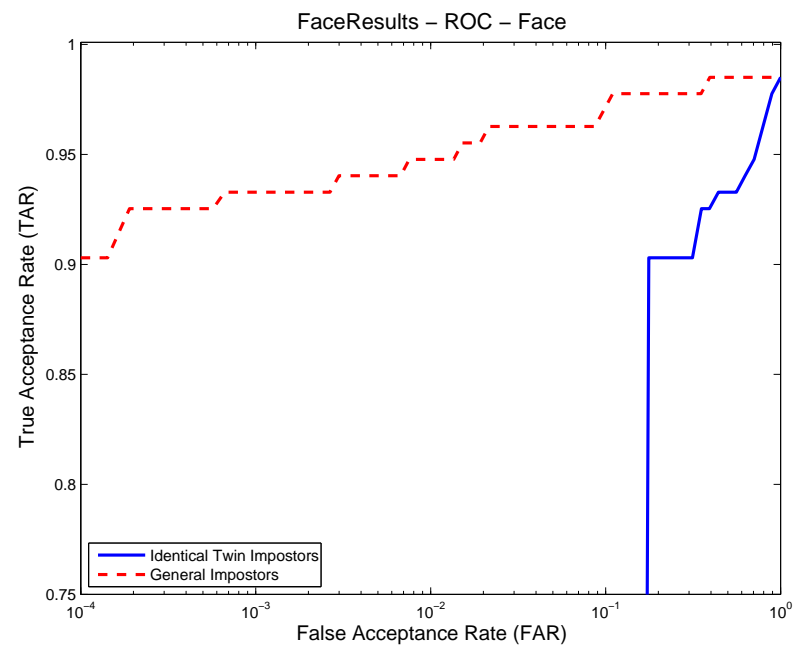

(c) Face

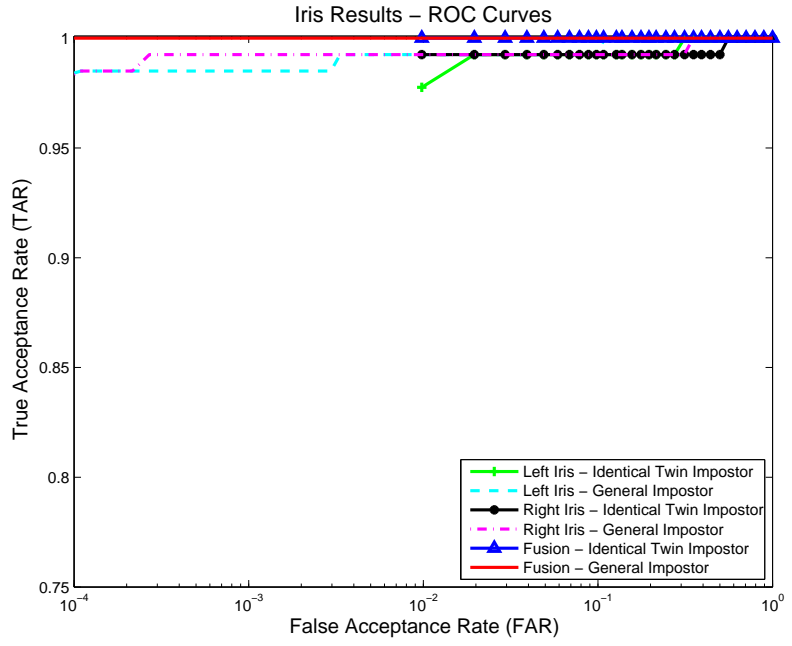

(b) Iris

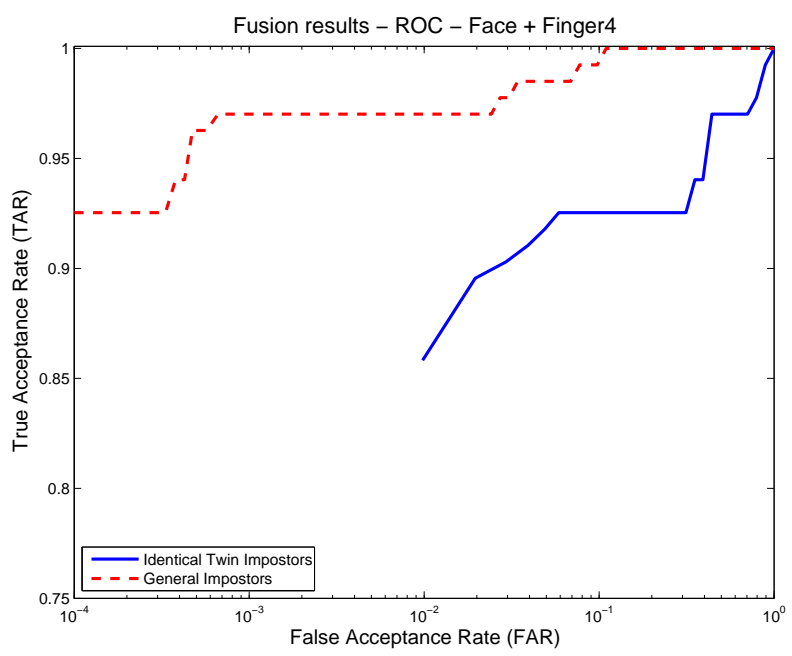

(d) Multimodal Fusion

Figure 7. ROC curves for fingerprint, iris and face, and a fusion example (face + finger 4 ). Due to the small number of identical twin impostor (102), FAR less than 1/102 cannot be estimated.

population and identical twins, multimodal biometric systems that combine different modalities (e.g. finger and face) show improvements in the performance on the general population compared to individual traits. However, the performance of multibiometric systems drop on the identical twin data compared to the highest performance modality that is being combined. This is because in the combination of finger and face matchers, the performance of the face matcher for identical twins is extremely poor.

To our knowledge, although multibiometric databases for twins have been collected before (the ten fingers and/or the two palmprints), no previous study has combined different units/modalities to study the multibiometric performance. We performed the multibiometric experiments and showed that the performance of a multibiometric system that uses different units of the same modality is improved compared to unimodal systems, reaching almost perfect accuracy on our database. Also, there have not been any previous studies of identical twin irises on a database this large. In addition, we have used commercial matchers for the face and fingerprint experiments, which are usually more accurate than in-house matchers (used in previous studies). Based on our experiments on this relatively small twin database, for example, we can conclude that the presence of identical twin data poses a real challenge to commercial face recognition systems. 


\section{ACKNOWLEDGMENTS}

Anil Jain's research was partially supported by WCU (World Class University) program through the National Research Foundation of Korea funded by the Ministry of Education, Science and Technology(R31-2008-00010008-0). Alessandra Paulino's research was supported by the Fulbright Program (A15087649) and the Brazilian Government through a CAPES Foundation/Ministry of Education grant (1667-07-6). This research was also supported in part by the National Basic Research Program of China (Grant 2004CB318110), Natural Science Foundation of China (Grant 60736018, 60702024), Hi-Tech Research and Development Program of China (Grant 2006AA01Z193, 2007AA01Z162), and the Chinese Academy of Sciences.

\section{REFERENCES}

[1] Martin, J. A., Kung, H.-C., Mathews, T. J., Hoyert, D. L., Strobino, D. M., Guyer, B., and Sutton, S. R., "Annual summary of vital statistics: 2006," Pediatrics , 788-801 (2008).

[2] UPI, "Identical twins guilty in bank fraud scheme," United Press International, 1756-1766 (September 24, 1986). Section: Domestic News.

[3] "Twin convicted in third rape trial," (March 22nd 2006). http://origin.foxnews.com/story/0,2933,188801,00.html.

[4] Jain, A. K., Prabhakar, S., and Pankanti, S., "On the similarity of identical twin fingerprints," Pattern Recognition 35, 2653-2663 (2002).

[5] Kong, A. W.-K., Zhang, D., and Lu, G., "A study of identical twins' palmprints for personal verification," Pattern Recognition 39, 2149-2156 (2006).

[6] Kodate, K., Inaba, R., Watanabe, E., and Kamiya, T., "Facial recognition by a compact parallel optical correlator," Measurement Science and Technology 13, 1756-1766 (2002).

[7] Ariyaeeinia, A., Morrison, C., Malegaonkar, A., and Black, S., "A test of the effectiveness of speaker verification for differentiating between identical twins," Science and Justice 48, 182-186 (2008).

[8] Daugman, J. and Downing, C., "Epigenetic randomness, complexity and singularity of human iris patterns," in [Proceedings of the Royal Society of London], 268, 1737-1740 (2001).

[9] Han, Y., Ryu, C., Moon, J., Kim, H., and Choi, H., "A study on evaluating the uniqueness of fingerprints using statistical analysis," in [Proceedings of the International Conference on Information Security and Cryptology], 467-477 (2005).

[10] Srihari, S. N., Srinivasan, H., and Fang, G., "Discriminability of fingerprints of twins," Journal of Forensic Identification 58(1), 109-127 (2008).

[11] Bronstein, A. M., Bronstein, M. M., and Kimmel, R., "Three-dimensional face recognition," International Journal of Computer Vision 64(1), 5-30 (2005).

[12] Lytle, J. M., "Biometric face scanner tells identical twins apart," (May 9th 2008). http://www.techradar.com/news/world-of-tech/future-tech/face-scanner-tells-identicaltwins-apart-363150.

[13] Patil, H. A. and Basu, T. K., "The teager energy based features for identification of identical twins in multilingual environment," in [Proceedings of the International Conference on Neural Information Processing], 333-337 (2004).

[14] Li, S. Z., ed., [Encyclopedia of Biometrics], vol. 1, Springer Science + Business Media (2009). Editorial Advisor: Anil Jain.

[15] Sun, Z. and Tan, T., "Ordinal measures for iris recognition," IEEE Transactions on Pattern Analysis and Machine Intelligence . in press.

[16] "Symwave: BioPrint USB Portable and Peripheral Solutions."

http://www.symwave.com/prod_biometric.shtml.

[17] "Neurotechnology: VeriFinger SDK." http://www.neurotechnology.com/verifinger.html.

[18] "Cognitec: FaceVACS - SDK." http://www. cognitec-systems.de/FaceVACS-SDK.19.0.html.

[19] "SDK-Beijing IrisKing Ltd. Co.." www.irisking.com/en/sdk.html. 